\title{
Downregulation of non-muscle myosin IIA expression inhibits migration and invasion of gastric cancer cells via the c-Jun $\mathrm{N}$-terminal kinase signaling pathway
}

\author{
TAO LIU*, YANWEI YE* , XIEFU ZHANG, ALI ZHU, ZHEN YANG, YANG FU, \\ CHONGQING WEI, QI LIU, CHUNLIN ZHAO and GUOJUN WANG
}

Department of Gastrointestinal Surgery, The First Affiliated Hospital of Zhengzhou University, Zhengzhou, Henan 450052, P.R. China

Received December 9, 2014; Accepted August 27, 2015

DOI: $10.3892 / \mathrm{mmr} .2015 .4742$

\begin{abstract}
Non-muscle myosin IIA (NMIIA) has been found to be overexpressed in gastric cancer tissues. However, the roles of NMIIA in the migration and invasion of gastric cancer cells have not yet been elucidated. The aim of the present study was to assess the effects of NMIIA knockdown on the migratory and invasive capacities of gastric cancer cells and to investigate the potential underlying mechanisms in vitro. First, the expression of NMIIA was assessed in gastric cancer tissues and non-cancerous tissues using western blot analysis. The expression levels of NMIIA protein in 51 out of 63 gastric cancer tissue specimens were higher compared to those in their matched non-tumoric gastric tissue specimens, and differences between the two groups were statistically significant $(\mathrm{P}<0.001)$. After downregulation of NMIIA using RNA interference, the migratory and invasive abilities of the SGC-7901 and MGC-803 gastric cancer cell lines were observed using a wound-healing assay and a Transwell assay, respectively. Knockdown of NMIIA significantly decreased the amount of wound closure as well as the number of cells which transgressed through the Matrigel $(\mathrm{P}<0.01)$. Finally, the levels of c-Jun N-terminal kinase (JNK), phosphorylated (p)-JNK, c-Jun and p-c-Jun were detected using western blot analysis in order to explore the association between NMIIA and the JNK signaling pathway. Knockdown of NMIIA decreased the levels of $\mathrm{p}$-JNK and p-c-Jun in the two gastric cancer cell lines $(\mathrm{P}<0.05)$. In conclusion, the present study
\end{abstract}

Correspondence to: Dr Xiefu Zhang, Department of Gastrointestinal Surgery, The First Affiliated Hospital of Zhengzhou University, 1 Jianshedong Road, Zhengzhou, Henan 450052, P.R. China

E-mail: xiefu_zhang@126.com

${ }^{*}$ Contributed equally

Key words: non-muscle myosin IIA, migration, invasion, gastric cancer, c-Jun $\mathrm{N}$-terminal kinase signaling pathway indicated that knockdown of NMIIA inhibited the migration and invasion of gastric cancer cells, which may be, at least in part, mediated via the JNK signaling pathway.

\section{Introduction}

Although the incidence of gastric cancer has decreased in the past 50 years in most developed countries, it remains one of the most important health issues in developing countries (1). According to the International Gastric Cancer Society, gastric cancer affects $>800,000$ people and accounts for 65,000 cancer-associated mortalities annually (2), therefore ranking as the fourth most common cancer type and the second leading cause of cancer-associated mortality $(3,4)$. Accumulating evidence has shown that aberrant cellular metabolism, involving multiple factors and steps, is a pivotal feature during tumorigenesis and cancer progression (5). However, the precise regulatory mechanisms underlying the development and progression of gastric cancer remain to be elucidated.

Non-muscle myosin IIA (NMIIA) belongs to the myosin II sub-family and is encoded by the MYH9 gene. It is an actin-based molecular motor that includes skeletal, cardiac, smooth muscle and non-muscular myosins $(6,7)$. Although the functions of NMIIA may be different in various cell types, interactions with actin microfilaments, microtubules, S100A4 as well as cadherin- and integrin complexes, have been identified, which may affect cellular activities, including tumor invasion (8).

Previous studies have indicated that NMIIA has a vital role in adhesion, invasion and migration of cancer cells, including breast cancer $(8,9)$, esophageal squamous cancer $(7)$, anaplastic large cell lymphoma (10) and gastric cancer $(6,11)$, as well as patient prognosis. The association between the overexpression of NMIIA and the progression as well as poor prognosis of gastric cancer has been clarified $(4,6)$; however, the signaling pathways of the involvement of NMIIA in gastric cancer have remained elusive.

The present study determined the levels of NMIIA expression in clinical gastric cancer tissues and matched non-tumorous gastric tissue specimens. Through in vitro Transwell and wound-healing assays, the present study 
assessed the invasive and migratory capacity of gastric cancer cells following MYH9 gene silencing by RNA interference (RNAi). In addition, the underlying molecular mechanisms of the roles of NMIIA in the invasion and migration of gastric cancer cells were explored. The present study provided insight into the role of NMIIA in gastric cancer progression.

\section{Materials and methods}

Patients and tissue specimens. Frozen clinical gastric cancer tissue specimens and matched non-tumorous gastric tissue specimens were collected from 63 gastric cancer patients from at the Department of Gastrointestinal Surgery (Zhengzhou, China). None of the patients received radiotherapy, chemotherapy or biotherapy prior to surgery. The present study was approved by the Ethics Committee of the First Affiliated Hospital of Zhengzhou University (Zhengzhou, China). Written informed consent was provided by the patients prior to commencement.

Cell lines and culture. The SGC-7901 and MGC-803 human gastric cancer lines were purchased from the Shanghai Institute of Cell Biology (Shanghai, China). The two cell lines were cultivated in RPMI 1640 (cat no. 21870-076; Gibco-BRL, Invitrogen Life Technologies, Inc., Carlsbad, CA, USA) supplemented with $10 \%$ fetal bovine serum (HyClone, Logan, UT, USA), 4 mM glutamine (cat no. 25030-149; Invitrogen Life Technologies), $100 \mathrm{U} / \mathrm{ml}$ penicillin (Sigma-Aldrich, St. Louis, MO, USA) and $100 \mu \mathrm{g} / \mathrm{ml}$ streptomycin (Sigma-Aldrich) in an incubator with a humidified atmosphere containing $5 \% \mathrm{CO}_{2}$ at $37^{\circ} \mathrm{C}(12)$.

Silencing of NMIIA. NMIIA isoform-specific small interfering (si)RNA and control siRNA were smart pools from Qiagen (Hilden, Germany). siRNA transfection was performed using HiPerfect transfection reagent (Qiagen) according to the manufacturer's instructions. Cells were plated in 12-well plates $72 \mathrm{~h}$ after transfection with $2 \mu \mathrm{M}$ siRNA. Following incubation for $24 \mathrm{~h}$, cells were harvested and subjected to subsequent experiments.

RNA extraction and reverse-transcription polymerase chain reaction $(P C R)$ analysis. Total RNA was extracted from minced tissues using TRIzol reagent (Invitrogen Life Technologies, Inc.), followed by cDNA synthesis using the TaqMan reverse transcription kit (cat. no. 4304134; Applied Biosystems; Thermo Fisher Scientific, Inc., Waltham, MA, USA). The primers used for the amplification of the cDNAs were as follows: NMIIA forward, 5'-AGAGCTCACGTG CCTCAACG-3' and reverse, 5'-TGACCACACAGA ACA GGCCTG-3'; $\beta$-actin forward, 5'-ATTGCCGACAGGATG CAGA-3' and reverse, 5'-GAGTACTTGCGCTCAGGA GGA-3' (Sangon Biotech, Shanghai, China). The PCR mixture (10 $\mu \mathrm{l}$ ) was comprised of $5 \mu \mathrm{l}$ TaqMan Fast Advanced Master Mix (Thermo Fisher Scientific, Inc.), $1 \mu 1$ cDNA (1:50 dilution) and $2 \mu \mathrm{l}$ of each forward and reverse primer $(1 \mathrm{mM})$. PCR was performed using the following cycling parameters: Denaturation at $95^{\circ} \mathrm{C}$ for $5 \mathrm{~min}$ in the first cycle and for $30 \mathrm{sec}$ in the second cycle, annealing at $57^{\circ} \mathrm{C}$ for $30 \mathrm{sec}$ and elongation at $72^{\circ} \mathrm{C}$ for $30 \mathrm{sec}$, with a final extension at $72^{\circ} \mathrm{C}$ for $5 \mathrm{~min}$.
The $\beta$-actin gene was used as an internal control. The PCR products were separated using agarose gel (Beyotime Institute of Biotechnology, Haimen, China) electrophoresis. Data were analyzed by $2^{-[\Delta \mathrm{Ct} \text { sample - } \Delta \mathrm{Ct} \text { control }]}$.

Western blot analysis. The expression of NMIIA in the frozen clinical tissue specimens and cultured cells at $24 \mathrm{~h}$ after siRNA transfection was examined using western blot analysis. The tissue specimens and cultured cells were homogenized and lysed with radioimmunoprecipitation assay lysis buffer (Beyotime Institute of Biotechnology), containing $100 \mathrm{mM}$ $\mathrm{NaCl}, 50 \mathrm{mM}$ Tris-HCl (pH 7.5), 1\% Triton X-100, 1mM EDTA, $10 \mathrm{mM}$ glycerophosphate, $2 \mathrm{mM}$ sodium vandate and protease inhibitor. Protein concentration was determined using a Micro-BCA protein kit (Pierce Biotechnology, Inc., Rockford, IL, USA), The protein (40 $\mu \mathrm{g} /$ lane) was then resolved by $12 \%$ SDS-PAGE (Beyotime Institute of Biotechnology). Following electrophoresis, the blots were transferred onto a polyvinylidene fluoride membrane (EMD Millipore, Billerica, MA, USA). The membranes were incubated with rabbit polyclonal NMIIA antibody (1:500; cat. no. ab24762; Abcam, Cambridge, UK) at $4^{\circ} \mathrm{C}$ overnight or anti- $\beta$-actin mouse monoclonal antibody $(1: 1,000$; cat. no. A1978; clone AC-15; Sigma-Aldrich) at $37^{\circ} \mathrm{C}$ for $2 \mathrm{~h}$. After washing with Tris-buffered saline containing Tween 20, the blots were visualized using an enhanced chemiluminescence kit (cat. no. sc-2048; Santa Cruz Biotechnology, Inc., Dallas, TX, USA). In addition, the levels of JNK and c-Jun in SGC-7901 cells were detected by western blot analysis. In this assay, antibodies against phosphorylated (p)-JNK (rabbit monoclonal; cat. no. 4668; clone 81E11; 1:1,500; Cell Signaling Technology, Inc., Danvers, MA, USA) and p-c-Jun (mouse monoclonal; cat. no. 2315; clone L70B11; 1:1,000; Cell Signaling Technology, Inc.), JNK (mouse monoclonal; cat. no. 610627; clone 37/pan-JNK/SAPK1; 1:1,000; BD Transduction Laboratories, Franklin Lakes, NJ, USA) and c-Jun (mouse monoclonal; cat. no. 610327; clone 3/Jun; 1:1,000; BD Transduction Laboratories) were used, which were incubated at $4^{\circ} \mathrm{C}$ overnight. $\beta$-actin (Sigma-Aldrich) were used. All procedures were repeated at least three times. Following antibody incubation, the membranes were washed with Tris-buffered saline containing Tween 20 (pH 7.4) three times. Following enhanced chemiluminescence, the blots were exposed to Kodak X-OMAT BT film (Kodak, Rochester, NY, USA). The bands were visualized using densitometry with Image-Pro Plus version 6.0 (Media Cybernetics, Inc., Rockville, MD, USA)

Transwell invasion assay. Briefly, $1 \times 10^{4}$ cells/well in serum-free medium were seeded into the upper chamber of a Transwell plate (cat no. 3422, Corning Inc., Corning, NY, USA) that was filter-coated with Matrigel (cat no. E1207; Sigma-Aldrich). At the same time, the bottom compartment of the chamber was filled with medium containing $10 \% \mathrm{FBS}$ as a chemoattractant. After $24 \mathrm{~h}$ of incubation at $37^{\circ} \mathrm{C}$ with $5 \%$ $\mathrm{CO}_{2}$, the cells remaining in the upper chamber were carefully removed using a cotton swab and the cells that had transgressed through the Matrigel and were located at the bottom of the membrane were fixed with $100 \%$ methanol and stained with hematoxylin. Quantification was performed by counting 
A

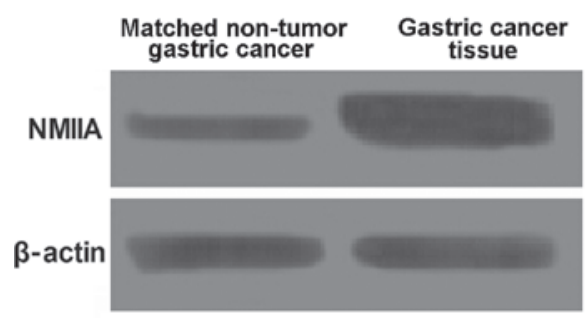

B

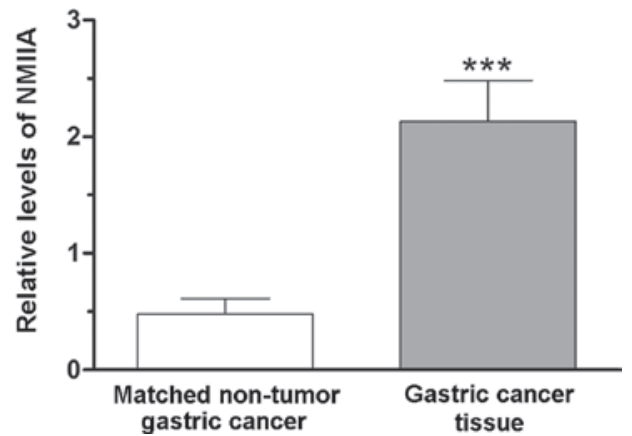

Figure 1. Expression levels of NMIIA in non-tumorous gastric tissues and gastric cancer tissues. (A) Representative western blot showing the levels of NMIIA in non-tumorous gastric tissue and gastric cancer tissue. (B) Relative levels of NMIIA in non-tumorous gastric tissues and gastric cancer tissues determined by densitometric analysis. Values are expressed as the mean \pm standard deviation of three experiments. ${ }^{* * *} \mathrm{P}<0.001$ vs. non-tumorous cancer tissues. NMIIA, non-muscle myosin IIA.

A

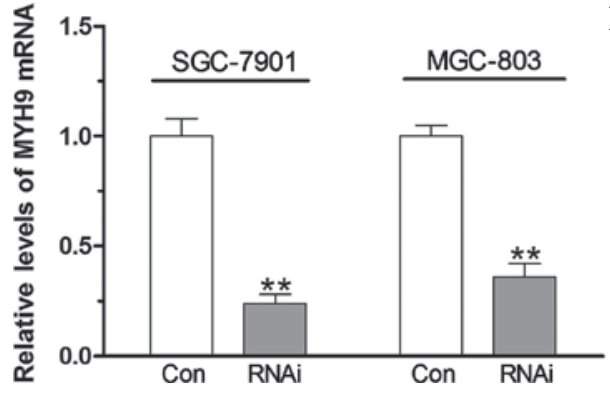

B

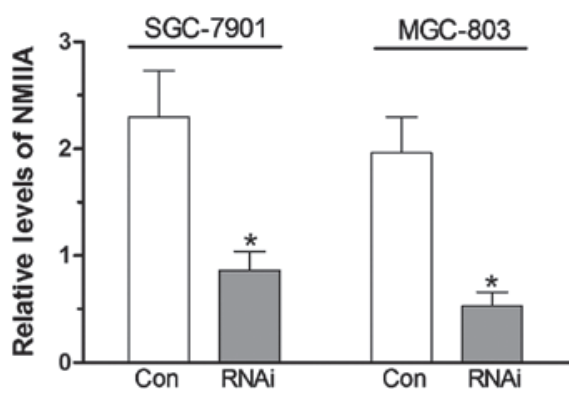

Figure 2. Inhibition of NMIIA by RNAi technology. (A) Relative levels of MYH9 mRNA in SGC-7901 and MGC-803 cells transfected with siRNA-MYH9. (B) Relative levels of NMIIA in SGC-7901 and MGC-803 cells transfected with siRNA-MYH9. Values are expressed as the mean \pm standard deviation of three experiments. ${ }^{*} \mathrm{P}<0.05 ;{ }^{* *} \mathrm{P}<0.01$, compared with the control. NMIIA, non-muscle myosin II; RNAi, RNA interference; MYH9, gene encoding NMIIA.

A

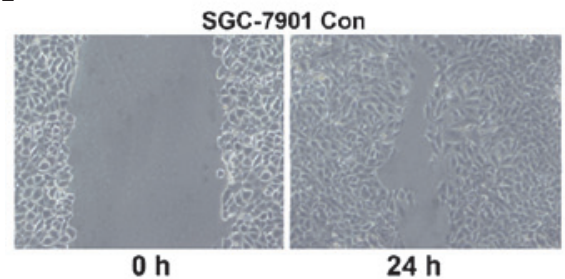

$\mathrm{Oh}$

D

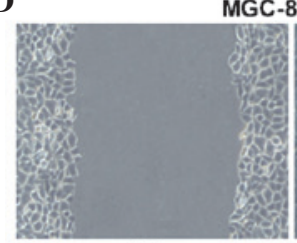

$\mathbf{O h}$
MGC-803 Con

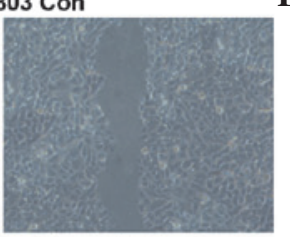

$24 \mathrm{~h}$
B

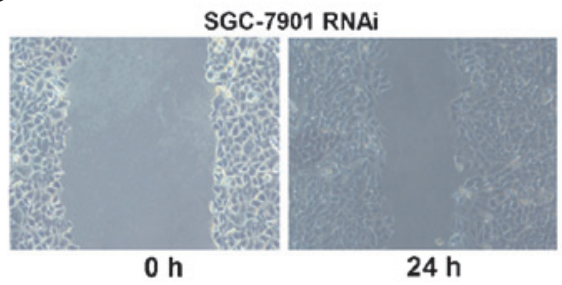

E

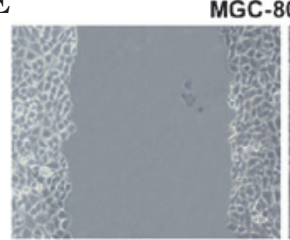

$\mathrm{Oh}$
C

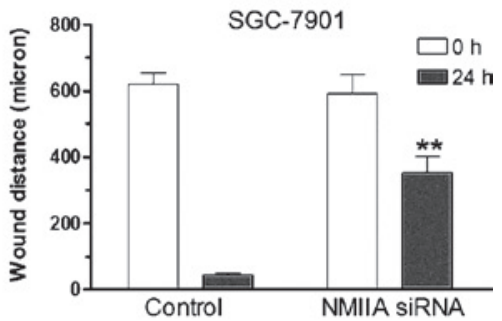

Figure 3. Suppression of NMIIA expression by RNAi blocks gastric cancer cell migration. (A) Wound distance of SGC-7901 cells at 0 and $24 \mathrm{~h}$ in wound-healing assay. (B) Wound distance of SGC-7901 cells subjected to MYH9-specific RNAi at 0 and 24 h in wound-healing assay. (C) Quantified results showing that the wound distance of SGC-7901 cells subjected to MYH9-specific RNAi was greater than that in the control group at $24 \mathrm{~h}$ in the wound-healing assay. (D) Wound distance of MGC-803 cells at 0 and $24 \mathrm{~h}$ in the wound-healing assay. (E) Wound distance of MGC-803 cells subjected to MYH9-specific RNAi at 0 and $24 \mathrm{~h}$ in wound-healing assay. Magnification, x100. (F) Quantified results showing that the wound distance of MGC-803 cells subjected to MYH9-specific RNAi was greater than that in the control group at $24 \mathrm{~h}$ in the wound-healing assay. Values are expressed as the mean \pm standard deviation of three experiments. ${ }^{* *} \mathrm{P}<0.01$ vs. control-transfected cells. NMIIA, non-muscle myosin IIA; RNAi, RNA interference; MYH9, gene encoding NMIIA.

the number of cells transgressed through the matrigel using an inverted microscope (GX41; Olympus Corporation, Tokyo, Japan) at 200x magnification.
Wound-healing assay. Cells were seeded into six-well tissue culture plates at $2 \times 10^{6}$ cells per well and grown in serum-free RPMI 1640 medium for $24 \mathrm{~h}$ to form a confluent monolayer. 
A

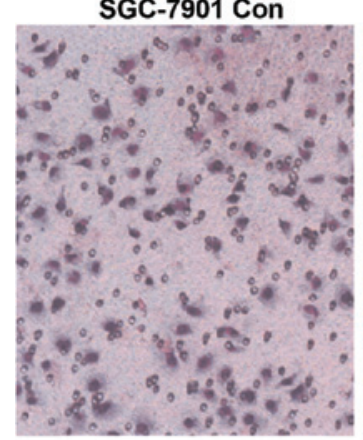

B

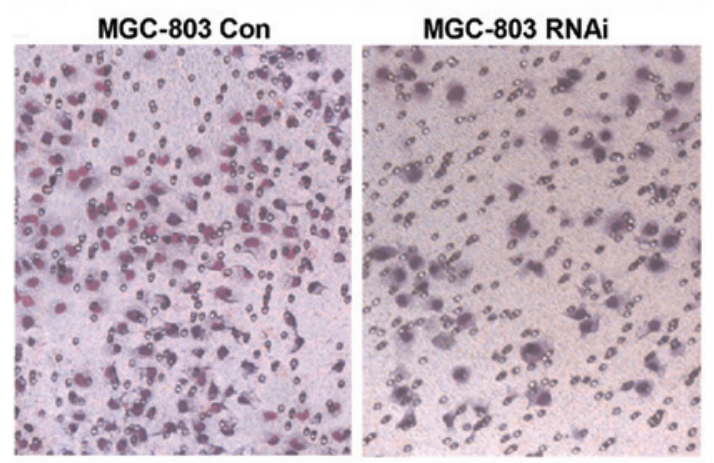

C

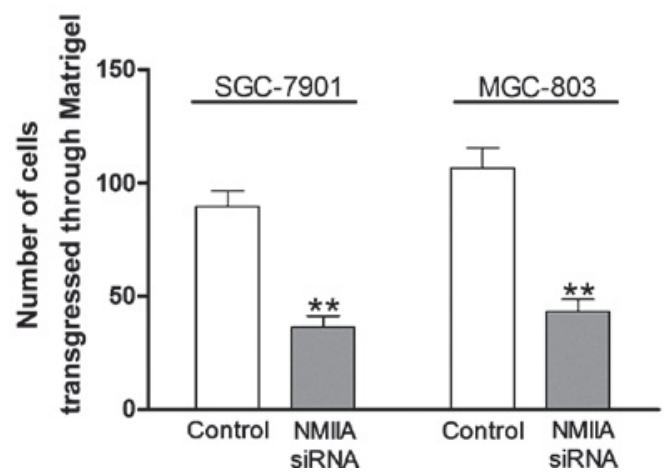

Figure 4. Downregulation of NMIIA expression inhibits invasion of gastric cancer cells. (A) Comparison of the number of SGC-7901 cells transfected with control siRNA or MYH9-specific siRNA transgressed through Matrigel. (B) Comparison of the number of MGC-803 cells transfected with control siRNA or MYH9-specific siRNA transgressed through Matrigel. Cells were stained with hematoxylin (magnification, x200). (C) Quantification of cell invasion demonstrated that RNAi reduced invasion of gastric cancer cells. Values are expressed as the mean \pm standard deviation of three experiments. ${ }^{* *} \mathrm{P}<0.01$ vs. control-transfected group. NMIIA, non-muscle myosin IIA; RNAi, RNA interference; MYH9, gene encoding NMIIA; siRNA, small interfering RNA; Con, control cells transfected with scramble siRNA.

A wound across the cell monolayer was created using a $100-\mu 1$ pipette tip (Sinoland, Qingdao, China). Cell migration into the wound area was then inspected under an IX70 inverted phase-contrast microscope (Olympus Corporation) at 100x magnification. The distance of wound closure was calculated for quantitative analysis.

Statistical analysis. Values are expressed as the mean \pm standard deviation. Student's t-test was used for comparing significant differences between the means of the two groups. Statistical analysis was performed using SPSS 13.0 software (SPSS, Inc., Chicago, IL, USA). A P-value from a two-tailed test of $<0.05$ was considered to indicate a statistically significant distance between values.

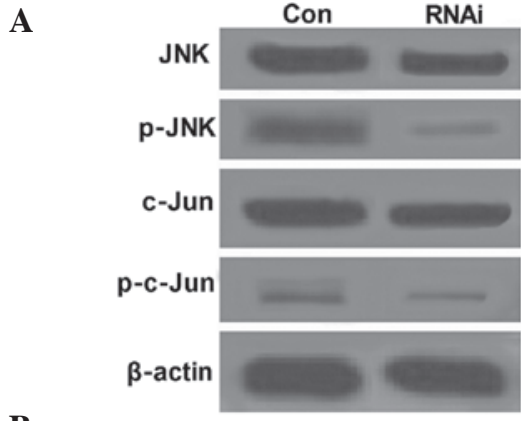

B

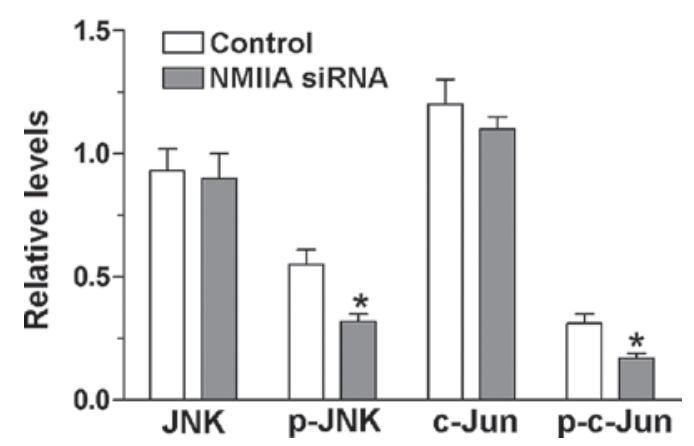

Figure 5. Downregulation of NMIIA expression blocks the JNK/c-Jun signaling pathway. (A) Representative western blots showing levels of JNK, p-JNK, c-Jun and p-c-Jun in SGC-7901 cells transfected with control siRNA or MYH9-specific siRNA. (B) Quantification of the blots showed that downregulation of NMIIA decreased the levels of p-JNK and p-c-Jun. Values are expressed as the mean \pm standard deviation of three experiments. ${ }^{*} \mathrm{P}<0.05$ vs. control. NMIIA, non-muscle myosin IIA; p-JNK, phosphorylated c-Jun N-terminal kinase; RNAi RNA interference; MYH9, gene encoding NMIIA; siRNA, small interfering RNA; Con, control cells transfected with scramble siRNA.

\section{Results}

Overexpression of NMIIA in gastric cancer. To investigate the levels of NMIIA expression in gastric cancer, clinical gastric cancer tissue specimens and matched non-tumorous gastric tissue specimens were subjected to western blot analysis. The results showed that the levels of NMIIA protein expression in 51 out of 63 gastric cancer tissue specimens were increased compared to those in their matched non-tumorous gastric tissue specimens. Statistical analysis showed that differences in NMIIA expression between gastric cancer tissues and matched non-tumorous gastric tissues were statistically significant $(\mathrm{P}<0.001)$ (Fig. 1).

Suppression of NMIIA expression by RNAi blocks gastric cancer cell migration. As shown in Fig. 2, the MYH9 gene was silenced in SGC-7901 and MGC-803 with specific siRNAs and the levels of NMIIA protein decreased compared with those in the control group $(\mathrm{P}<0.05)$. A wound-healing assay was performed to assess the migration capacity of gastric cancer cells. As shown in Fig. 3, knockdown of NMIIA inhibited the migratory capacity of SGC-7901 and MGC-803 cells, as indicated by a decreased amount of wound closure of gastric cancer cells following knockdown of the MYH9 gene $(\mathrm{P}<0.01)$, indicating that NMIIA is involved gastric cancer cell migration.

Downregulation of NMIIA expression inhibits the invasion of gastric cancer cells. A Matrigel invasion assay was performed 
to observe the effects of NMIIA knockdown on the invasive capacity of gastric cancer cells. As shown in Fig. 4A and B, knockdown of NMIIA inhibited the number of SGC-7901 and MGC-803 cells transgressing though the Matrigel. Quantification of the results showed that NMIIA knockdown significantly reduced the invasive capacities of the two cell lines $(\mathrm{P}<0.01)$ (Fig. 4C).

NMIIA is an activator of the JNK signaling pathway. The levels of p-JNK and p-c-Jun in SGC-7901 cells were detected using western blot analysis prior to and following knockdown of NMIIA expression by siRNA. The results showed that the levels of p-JNK and p-c-Jun in SGC-7901 cells were high. However, after inhibition of NMIIA expression by siRNA, the levels of p-JNK and p-c-Jun were significantly decreased $(\mathrm{P}<0.05)$, which indicated that NMIIA is an activator of JNK and c-Jun (Fig. 5).

\section{Discussion}

Gastric cancer is one of the most frequent cancer types in the world (13). The mechanisms responsible for the occurrence, development and prognosis of gastric cancer have been investigated from various perspectives. For instance, Wang et al (3) investigated the underlying regulatory signaling pathways in gastric cancer by integrating gene expression profiles and transcriptional regulatory element databases, showing that the five transcription factors hypoxia-inducible factor-1 $\alpha$, nuclear factor- $\kappa \mathrm{B} 1$, breast cancer 1 , signal transducer and activator of transcription (STAT)3 and STAT1 were able to regulate 82 differentially-expressed genes in gastric cancer. In addition, these genes formed 95 regulation modes, among which MMP1, TIMP1, TLR2, FCGR3A, IRF1, FAS and TFF3 were central genes that were simultaneously regulated by at least two of these five transcription factors, and were associated with hypoxia, inflammation and immune disorders. In addition, a recent study revealed that the expression of CXC motif receptor 1 and 2 proteins promoted MMP-9 expression by activating JNK/c-Jun and extracellular signal-regulated kinase (ERK)/Ets-1 pathways, resulting in a more aggressive phenotype in gastric cancer cells (12). The present study examined the effects of NMIIA on the invasion and migration of gastric cancer cells, based on previous evidence showing that NMIIA was overexpressed in certain types of cancer $(7,9,10)$. The results of the present study showed that NMIIA was overexpressed in gastric cancer tissues, which was consistent with the results of a previous study (6).

JNK, a member of the mitogen-activated protein kinase family that regulates a range of pathological processes involved in tumor and brain development and neurological disorders (14), is encoded by three genes: JNK1 and 2, which are ubiquitously expressed, and JNK3, which is restricted to the testis, heart and brain $(15,16)$. The functions of JNK isoforms in diseases have been most thoroughly demonstrated in cancer (14). According to previous studies, JNK1 deficiency significantly decreased hepatocellular carcinoma in a mouse model (17), while JNK2 was shown to act as a tumor promoter in skin cancer formation (18). A number of studies have explored the JNK signaling pathway in gastric cancer. When SGC-7901 cells were treated with vitamin $\mathrm{E}$ succinate, transforming growth factor- $\beta$ was activated, which in turn increased the activity of JNK, which then induced c-Jun phosphorylation; finally, p-c-Jun initiated apoptosis of gastric cancer cells (19). Similarly, the antioxidant analogue $\alpha$-tocopheryl succinate induced apoptosis by activating ERK1/2 and JNK via c-Jun in the gastric cancer cell line SGC-7901 (20). In addition, the fact that the specific JNK inhibitor SP-600125 inhibited cell viability, induced apoptosis and caused cell cycle arrest in gastric cancer cells was most likely associated with its inhibition of JNK2 phosphorylation, which attenuated the JNK signaling pathway (21). The present study found that knockdown of NMIIA inhibited the migration and invasion of gastric cancer cells, while simultaneously decreasing the protein levels of JNK and c-Jun as well.

It is known that JNK is able to phosphorylate c-Jun on serines 63 and 73 at the N-terminal activating sites, which leads to increased stability of c-Jun and an increase in its transactivation potential and DNA-binding affinity (22-24). The results of the present study indicated that NMIIA inhibited the activation of JNK, resulting in the inhibition of c-Jun phosphorylation, which, in turn, attenuated the migratory and invasive capacities of gastric cancer cells. In fact, the JNK signaling pathway is involved in the migration and invasion of cancer cells, which has been demonstrated by various studies on the basis of a range of in vivo and in vitro experimental models. For instance, isoliquiritigenin decreases the phosphorylation of JNK and c-Jun and certain other regulatory factors, which inhibits the migration, adhesion and invasion of prostate cancer cells.

In conclusion, the present study demonstrated that NMIIA was overexpressed in gastric cancer and knockdown of NMIIA by RNAi inhibited the migration and invasion of gastric cancer cells in vitro, which may proceed via the JNK signaling pathway. The present study may be useful for the development of novel strategies for the clinical control of gastric cancer metastasis.

\section{References}

1. Herszényi L and Tulassay Z: Epidemiology of gastrointestinal and liver tumors. Eur Rev Med Pharmacol Sci 14: 249-258, 2010.

2. Compare D, Rocco A and Nardone G: Risk factors in gastric cancer. Eur Rev Med Pharmacol Sci 14: 302-308, 2010.

3. Wang J, Ni Z, Duan Z, Wang G and Li F: Altered expression of hypoxia-inducible factor-1 $\alpha$ (HIF-1 $\alpha)$ and its regulatory genes in gastric cancer tissues. PLoS One 9: e99835, 2014.

4. Liu X, Liu Q, Fan Y, Wang S, Liu X, Zhu L, Liu M and Tang H: Downregulation of PPP2R5E expression by miR-23a suppresses apoptosis to facilitate the growth of gastric cancer cells. FEBS Lett 588: 3160-3169, 2014.

5. DeBerardinis RJ, Lum JJ, Hatzivassiliou G and Thompson CB: The biology of cancer: Metabolic reprogramming fuels cell growth and proliferation. Cell Metab 7: 11-20, 2008.

6. Liu D, Zhang L, Shen Z, Tan F, Hu Y, Yu J and Li G: Clinicopathological Significance of NMIIA overexpression in human gastric cancer. Int J Mol Sci 13: 15291-15304, 2012.

7. Xia ZK, Yuan YC, Yin N, Yin BL, Tan ZP and Hu YR: Nonmuscle myosin IIA is associated with poor prognosis of esophageal squamous cancer. Dis Esophagus 25: 427-436, 2012.

8. Derycke L, Stove C, Vercoutter-Edouart AS, De Wever O, Dollé L, Colpaert N, Depypere H, Michalski JC and Bracke M: The role of non-muscle myosin IIA in aggregation and invasion of human MCF-7 breast cancer cells. Int J Dev Biol 55: 835-840, 2011.

9. Betapudi V, Licate LS and Egelhoff TT: Distinct roles of nonmuscle myosin II isoforms in the regulation of MDA-MB-231 breast cancer cell spreading and migration. Cancer Res 66: 4725-4733, 2006. 
10. Lamant L, Gascoyne RD, Duplantier MM, Armstrong F, Raghab A, Chhanabhai M, Rajcan-Separovic E, Raghab J, Delsol G and Espinos E: Non-muscle myosin heavy chain (MYH9): A new partner fused to ALK in anaplastic large cell lymphoma. Gene Chromosomes Cancer 37: 427-432, 2003.

11. Liang S, He L, Zhao X, Miao Y, Gu Y, Guo C, Xue Z, Dou W, $\mathrm{Hu}$ F, Wu K, et al: MicroRNA let-7f inhibits tumor invasion and metastasis by targeting MYH9 in human gastric cancer. PLoS One 6: e18409, 2011.

12. Li Z, Wang Y, Dong S, Ge C, Xiao Y, Li R, Ma X, Xue Y, Zhang Q, Lv J, et al: Association of CXCR1 and 2 expressions with gastric cancer metastasis in ex vivo and tumor cell invasion in vitro. Cytokine 69: 6-13, 2014.

13. Matsuoka J, Yashiro M, Sakurai K, Kubo N, Tanaka H, Muguruma K, Sawada T, Ohira M and Hirakawa K: Role of the stemness factors sox 2 , oct3/4 and nanog in gastric carcinoma. J Surg Res 174: 130-135, 2012.

14. Davies C and Tournier C: Exploring the function of the JNK (c-Jun N-terminal kinase) signalling pathway in physiological and pathological processes to design novel therapeutic strategies. Biochem Soc Trans 40: 85-89, 2012.

15. Johnson GL and Nakamura K: The c-jun kinase/stress-activated pathway: Regulation, function and role in human disease. Biochim Biophys Actas 1773: 1341-1348, 2007.

16. Ma J, Zhang L, Han W, Shen T, Ma C, Liu Y, Nie X, Liu M, Ran Y and Zhu D: Activation of JNK/c-Jun is required for the proliferation, survival and angiogenesis induced by EET in pulmonary artery endothelial cells. J Lipid Res 53: 1093-1105, 2012.

17. Hui L, Zatloukal K, Scheuch H, Stepniak E and Wagner EF: Proliferation of human HCC cells and chemically induced mouse liver cancers requires JNK1-dependent p21 downregulation. J Clin Invest 118: 3943-3953, 2008.
18. Chen N, Nomura M, She QB, Ma WY, Bode AM, Wang L, Flavell RA and Dong Z: Suppression of skin tumorigenesis in c-Jun NH2-terminal kinase-2-deficient mice. Cancer Res 61: 3908-3912, 2001.

19. Wu K, Liu BH, Zhao DY and Zhao Y: Effect of vitamin E succinate on expression of TGF-beta1, c-Jun and JNK1 in human gastric cancer SGC-7901 cells. World J Gastroenterol 7: 83-87, 2001.

20. Zhao Y, Zhao X, Yang B, Neuzil J and Wu K: $\alpha$-Tocopheryl succinate-induced apoptosis in human gastric cancer cells is modulated by ERK1/2 and c-Jun N-terminal kinase in a biphasic manner. Cancer Lett 247: 345-352, 2007.

21. Xia HH, He H, De Wang J, Gu Q, Lin MC, Zou B, Yu LF, Sun YW, Chan AO, Kung HF and Wong BC: Induction of apoptosis and cell cycle arrest by a specific c-Jun NH2-terminal kinase (JNK) inhibitor, SP-600125, in gastrointestinal cancers. Cancer Lett 241: 268-274, 2006.

22. Qi X, Pramanik R, Wang J, Schultz RM, Maitra RK, Han J, DeLuca HF and Chen G: The p38 and JNK pathways cooperate to trans-activate vitamin D receptor via c-Jun/AP-1 and sensitize human breast cancer cells to vitamin $\mathrm{D}(3)$-induced growth inhibition. J Biol Chem 277: 25884-25892, 2002.

23. Schroeter H, Spencer J, Rice-Evans C and Williams R: Flavonoids protect neurons from oxidized low-density-lipoprotein-induced apoptosis involving c-Jun N-terminal kinase (JNK), c-Jun and caspase-3. Biochem J 358: 547-557, 2001.

24. Wu K, Zhao Y, Li GC and Yu WP: c-Jun N-terminal kinase is required for vitamin $\mathrm{E}$ succinate-induced apoptosis in human gastric cancer cells. World J Gastroenterol 10: 1110-1114, 2004. 